\title{
General Model for Adequate Cloud Service Selection using Decision Making Methods
}

\author{
O. Pantelić, A. Pajić, A. Nikolić
}

\section{Ognjen Pantelić́, Ana Pajić}

Department of Information Systems

Faculty of Organizational Sciences, University of Belgrade

Jove Ilica 154, 11000 Belgrade, Serbia

*Corresponding author: pantelico@fon.bg.ac.rs

ana.pajic@fon.bg.ac.rs

\author{
Ana Nikolić \\ Novomatic Lottery Solutions \\ Djordja Stanojevica 12, 11070 Novi Beograd, Serbia \\ anikolic@novomaticls.com
}

\begin{abstract}
Cloud Computing (CC) is a technology that surely brings innovations in today's business world, more and more companies around the world are widely incorporating this technology into their businesses. From a technical, as well as organizational point of view transferring enterprise IT to the Cloud is a complex task. Various factors have to be taken into consideration in order to make a right choice when moving IT services to the Cloud.

The goal of this paper is to identify and to discuss in detail all factors that influence organization's decision to adopt Cloud. General model for Cloud adoption, introduced in Pantelić et al. [13] ${ }^{a}$, consists of the key factors driving the organizational benefits when moving to the Cloud. The purpose of the model is to support decision makers in evaluating the benefits, risks and costs of using Cloud Computing. In this paper the general model is extended with two new aggregation methods for harmonization of alternatives rankings in a group decision process. We present the results of two new methods using the method results from previous research [13], as rank inputs, into an aggregate (group) preference. The idea is to find consensus ranking that minimizes disagreement among previous methods results.

There were no strong differences between the results of performed methods. The results have shown that Software as a service model and Storage as a service model dominated according to not just arithmetic-mean method, but also to geometric-mean method.

Keywords: cloud adoption, general model, IT investments, cloud services, multicriteria methods.

\footnotetext{
${ }^{a}$ Reprinted (partial) and extended, with permission based on License Number 3958430574606 C)[2016] IEEE, from "Computers Communications and Control (ICCCC), 2016 6th International Conference on".
}

\section{Introduction}

During the past decade, many enterprises have shifted their focus towards the use of Cloud Computing (CC) as a viable option to reduce costs and to improve IT and business agility. The Cloud Computing model provides almost unlimited computing resources for users which are taken on demand and pay per use. It represents an extension of distributed computing and grid computing technology, which aims to deliver a variety of services over the Internet via Cloud providers. CC offers a lot of advantages to the businesses of all sizes. By moving business to the Cloud, IT decision makers can take advantage of highly efficient Cloud infrastructure 
which reduces energy consumption considerably and they are freed of problems related to the technological issues of installing and maintaining the IT. However, there are many uncertainties with regard to the actual realization of these benefits, particularly around the cost savings and the data security. Despite many Cloud applications are currently widely and successfully used, the growth of CC has slowed down. According to [6], the technical aspect of security in the Cloud is one of the main reasons for the slowing down in the growth of CC. Reference [15] underlined security, interoperability, and portability as major obstacles to broader Cloud adoption.

This research is an extension of [13] (doi: 10.1109/ICCCC.2016.7496751). The general model for Cloud adoption introduced in [13] is extended with two new methods for group decision making. Goal of the paper is to present approaches to creating the preference structure of alternatives by assigning ordinal ranks to the alternatives. Paper presents results of two new methods using the method results from our previous research, as rank inputs, into an aggregate (group) preference. The idea is to find consensus ranking that minimizes disagreement among previous methods results. In addition, we discussed in detail key factors driving Cloud adoption.

The paper is organized as follows. Section 2 points some background related to Cloud Computing and presents in detail advantages and disadvantages of different Cloud services. In section 3 , we introduce a general model of Cloud adoption decision process with multi-criteria decision making methods as one of the key factors. Detailed analysis of relevant factors is reported. The purpose of the model is to support decision makers in evaluating the benefits, risks and costs of using CC. The model can be used as the guideline for enterprises that are considering moving their IT services to the Cloud. Our results from implementation of multi-criteria decision making methods, in particular Analytics Hierarchy Process (AHP), Iterative Compromise Ranking (ICOR) and Multi- Attribute Utility Theory (MAUT), are given in Section 4.1, and the results of new preference aggregation methods are given in Section 4.2. The last section contains our concluding remarks and ideas for future work.

\section{Cloud Computing Service Models}

There are many definitions of CC. A definition of CC by NIST (National Institute of Standards and Technology) is "a model for enabling ubiquitous, convenient, on-demand network access to a shared pool of configurable computing resources (e.g., networks, servers, storage, applications, and services) that can be rapidly provisioned and released with minimal management effort or service provider interaction" [10]. Reference [3] characterize CC as a pay per use model which offering computing, storage and software "as a service". An important aim of this technology is delivering computing as a utility.

$\mathrm{CC}$ has always been divided into three broad service categories: Infrastructure as a Service (IaaS), offers virtualized computation, storage and communication resources to the customers on demand, Platform as a Service (PaaS), provides an environment for developing applications using languages, services and tools supported by provider, and finally Software as a Service (SaaS), provides to the consumer applications and services that run on the providers infrastructure. As new Cloud providers enter the market, even more Cloud service models are available today. Many additional models can be found in literature [16]. These service models are Network as a Service, Storage as a Service, Database as a Service, Security as a Service, Integration as a Service, Management as a Service, Testing as a Service, Information as a Service, Communication as a Service and Monitoring as a Service. They share many similarities but have their own distinct differences as well. We will analyze in more detail below the characteristics of these models.

Cloud service models describe how cloud services are made available to clients. The most widely used Cloud service model is SaaS that offers an on demand online software subscription. SaaS provides significant efficiencies in cost and delivery by removing the need for technical staff 
to install, manage and upgrade software. Moreover, it reduces the cost of licensing software. The consumer does not control the underlying Cloud infrastructure and IT operational functions, which cause important concern related to security, performance and service availability. Some software customization is often available, but this is more limited than with PaaS and IaaS models. With PaaS, consumers have control over the deployed applications while the service provider delivers the computing platform at predictable service level and cost. The platform consists of required software tools and it is hosted on the hardware infrastructure of the service provider. In this model, it is not necessary to hire people to maintain the platform and hardware infrastructure. Benefits for using PaaS include the ability to eliminate cost of upkeep for hardware and the consumers can concentrate on building software. The downside is that the developers must work within the constraints of the platform which may cause data portability and application interoperability issues. On the other side, consumers have more real control over their infrastructure with IaaS than with PaaS or SaaS model. IaaS model allows automated deployment of servers, processing power, networking and storage. Consumers have direct access to their infrastructure resources, just as they would with traditional infrastructure but optimize infrastructure spend. The downside is that some applications may not run on available infrastructure of service provider due to their very specific hardware requirements. "Compared to IaaS offerings, applications riding on PaaS deliver better performance due to the intrinsic cloud support for the programming platform" highlighted Buyya et al. in [3]. Core datacenter infrastructure consists of storage, servers, the network, and management tool for infrastructure maintenance and monitoring. Each of these components has created a separate market niche. It enables the design of efficient in-network services. Network as a Service is not a new concept, but a new business model for delivery efficient in-network services [5]. It enables the design of efficient in-network services. However, the same concern about the Cloud provider's ability to guarantee high service availability appears. Storage as a Service allows consumers to manage data storage with no need for IT staff and to align IT spends with data consumption. With Storage as a Service, disaster recovery of data in the Cloud is offered. The drawbacks are long latencies and limited bandwidth [14]. Database as a Service delivers database functionality as a service and consumers can deploy new databases quickly, securely and cheaply. The downside is a lack of control over network performance issues [16]. On the other side, Gartner is predicting a growth of the Cloud-based security service market [11]. Security as a Service is data and application protection solution, which is usually included in SaaS model. Businesses employ Integration as a Service in order to share data between systems as well as with one or more external applications in real-time. The ultimate goal is the process improvement, to gain agility and automate business processes. Besides, Management as a Service model offers a suit of SaaS based applications to ensure cost-effective IT management operations. Consumers can easily analyze, monitor and evaluate performance metrics to ensure the optimization of IT resources [18]. The Monitoring as a Service offerings are focused on monitoring the insights of IT infrastructure assets. It provides the complete overview of the infrastructure, servers, networks, storage as well as the applications. Privacy concerns are still the main obstacles in adapting this model. Communication as a Service is known as a collection of different vendors' services that facilitate enterprise communications. The core concept of this model is that using this service is very convenient. Furthermore, Information as a Service integrates the information to provide real-time and high quality information about a business entity [17]. The last model is Testing as a Service which delivers the on-demand test execution. It is most suitable for test executions that do not require in-depth knowledge of the system. 


\section{General Model Elements}

There are many factors that determine which Cloud service model is right for the specific organizational needs. A review of the literature reveals that factors impacting Cloud adoption tend to be psychological as well as technical. The decision on migrating IT services to the Cloud should take into consideration a variety of socio-technical factors. It is not sufficient to examine simply the cost of deploying their IT systems on the Cloud.

In [13] the general model for Cloud adoption was briefly presented, aiming to support the Cloud adoption decision process. The model consists of seven key factors driving the organizational benefits when moving to the Cloud. Decision makers can use the model to evaluate the benefits, risks and costs of using Cloud. It is important that decision makers view Cloud adoption project from different perspectives in order to fully understand Cloud services and their various pros and cons. As most of the pros and cons are unquantifiable, making the decision about Cloud services today's biggest concern. It is important to notice a list of the major factors that weight into the decision. Once decision makers understand the key factors driving Cloud adoption, the next challenge is to select a most suitable Cloud service to migrate their IT services.

Therefore, in this section we thoroughly evaluate key factors that influence on organization decision to adopt Cloud Computing. The starting point must be to determine a company's objectives. It is important to consider what the company wants to achieve by migrating IT services to the Cloud. Ideally any company wants to increase profit, while decreasing cost. Cloud computing has the ability not only to decrease costs, but also to run the business more effectively. It can reduce the cost of equipment, energy, and infrastructure administration and management, needed to run the same business. Moreover, the business can remain current on technological advances. The company will be able to invest in new projects for direct business benefits, which eventually increases business value. Although Cloud Computing has many advantages over traditional techniques, it does not necessarily mean that Cloud will improve the business. Cloud migration can result in a considerable amount of organizational changes. For example, system administrators will no longer have complete control of IT infrastructure and services. In order to take advantages of the Cloud, the company should look to align Cloud service with business requirements and objectives. From an enterprise perspective, flexibility, scalability, and business continuity are also important besides cost. One of the most significant barriers to adoption is security, mainly the migration of critical applications and sensitive data to public cloud environment [8]. Perhaps organizations will not completely outsource their back-end computing resources and activities to Cloud provider. Rather, they will engage in a partnership with more than one Cloud provider and will establish heterogeneous computing environments.

The influence of Cloud services on global economy and environment is also significant. Cloud Computing seems to be a new model for doing business with a potential to change global industries' business models. Investing in Cloud globally will create new jobs and economic opportunities. It will allow developing economics to become more competitive driving more investments. Additionally, there is in fact a risk of IT sector job loss from investing in Cloud. For example, companies are more likely to move jobs related to maintaining infrastructure to the Cloud. IT departments will not need server engineers, systems administrators, or data center managers, however, they will still need good network engineers, IT managers and business analysts to support applications. Traditional roles will change and expand in development flexible application that suits Cloud environment. There will be new opportunities for IT staffs if they adjust their skills and focus more on systems integration, system security and data privacy. The organization will be more collaborative as employees can work from any location, any time. Furthermore, adopting cloud technologies requires changes in organizational culture and people's perception of new technologies. Employees may be fear on how new technologies will affect their work, which 
can result in employees resistance. Thus, employees' perception of Cloud benefits is possible barrier to Cloud adoption.

Cloud services also reducing IT sector's carbon footprint. Cloud providers operate in a massive scale, so energy consumption, the number of harmful substances and waste is significantly lower compared to the traditional in-house business. Since Cloud Computing paradigm is based on sharing configurable computer resources by multiple users, the number of equipment, facilities, and resources for business can be greatly reduced or completely eliminated. This way of doing business can certainly lead to a more sustainable IT infrastructure and processes. In July 2011, The Carbon Disclosure Project commissioned a study that examined financial and carbon benefits of Cloud [4]. A total of eleven organizations in the United States that have been using Cloud Computing for at least two years participated in the study. Detailed case studies revealed a 50 percentage reduction of carbon in 2020 with adopting Cloud technologies versus no Cloud at all. The potential carbon reductions of 85.7 million tons per year by 2020 for multi billion dollars United States companies is comparable to the annual emissions from 16.8 million passenger vehicles. The report shows that organizations can achieve an annual energy use reduction of 12.3 billion dollars by 2020 .

The next step is CC evaluation based on financial metrics. As already mentioned, CC saves money but it might lead to financial loss due to security risks, possible data access problems, etc. Researchers recommend using several financial metrics to evaluate how well Cloud would be beneficial for enterprises. Decision makers should be able to use these metrics to assess what Cloud offering should best meet business and technical requirements. Metrics are used to understand the factors needed for clear distinction between two and more different Cloud offerings. These metrics are used for measurement of the business value of IT. There are direct and indirect financial metrics. Direct metrics refers to indicators that measure financial gain or loss at limited or no distance from the production functions, such as a metric used to measure investment performance. By contrast, indirect methods measure general business and IT performance. Most commonly used metrics are Cost Benefit Analysis (CBA), Return on investment (ROI), and Total cost of ownership (TCO) [9].

Cost Benefit Analysis is widely used direct financial metric which simply calculate the value of the benefits, and subtracts the financial cost associated with it. The payback method is built into the analysis. It measures the length of time required to regain the benefits of investment to repay its cost. The period of time could be one month, one year, or three years mostly. It lacks of the time value of money (TVM) function, which is considered reliable for investments whose benefits expected to be received over longer periods of time. On the other side, Net present value (NPV) analysis considers the time value of money through the use of discount rate. Discount rate is here the most critical decision variable used to define the present value of an investment. The present value of an investment is always less than or equal to the future value at the end of determined period of time. Academics' and practitioners' recommendations regarding discount rate are ranged from 1 to 15 percent. Net benefits are simple calculated by the sum of benefits discounted at the discount rate minus cost. If Cloud Computing project has a positive NVP value then it is expected to be a good decision. According to the Investopedia, Return on investments (ROI) is a cash flow metric used to compare the efficiency of a number of different investments. When risks and other factors between the choices are equal, the investment with the higher ROI is considered the better decision. Most forms of ROI analysis compare the benefits of investment with the cost of the investment. The result is represented as a ratio or percentage. A positive ROI result means that investment returns is higher than cost. If Cloud business case scenario has a positive ROI, it can be considered as investment with net gain. A simple ROI equation is not adjusted for the time value of money. Therefore, it cannot be trusted to measure returns over long periods of time. Investing in Cloud Computing can deliver 
significant long term benefits. It is good practice to use ROI metric discounted for present value. The result will be considerably less than the unadjusted ROI. Cloud Computing investment will be still worthwhile, if the discounted ROI result is higher than company's minimum acceptable rate of return.

Up to this point, we have discussed the three most common direct metrics. In the following, we introduced Total cost of ownership (TCO) and Time to market as indirect metrics. Measuring the business impact of IT functions begins with comprehensive TCO methodology. TCO tries to quantify all tangible expenses, as well as some intangible ones that can be assigned a monetary value. Total costs are a critical component of the IT value equation, and TCO analysis includes the costs of deploying an IT product across the product's whole life cycle. For Cloud technologies, expenses may depend on selected Cloud service and selected Cloud provider. There are costs associated with Cloud license and maintenance fees, integration process, and employee training. At the same time, Cloud can reduce cost for software and hardware upgrades, infrastructure cost, energy cost, labour costs, etc. Many cloud providers will reveal a TCO analysis to show the overall costs and benefits associated with migrating to their Cloud platform. Hence, Cloud service evaluation can be done by comparing TCO of Cloud solution with TCO of traditional IT departments within enterprise [9]. For IT department, the agility to deploy new applications and enter new markets is important key performance indicator. It can be measured by Time to Market (TTM) metrics which measures the length of time to lunched new product or service. Lower TTM leads to an increase in the company's competitive advantage. As TTM increases, the risk of losing customers also increases. With Cloud Computing, speed to market can be reduced while significantly reducing the cost.

However, it is no longer sufficient to measure only financial performance [12]. Instead, it is necessary to use multi-criteria decision analysis methods to determine the value of intangible assets. By using these methods, decision makers can rate and apply weights to quality attributes of different Cloud service models, based on their preferences. The trend is to combine two or more methods to make up for shortcomings in any single particular method. Analytic Hierarchy Process (AHP), Iterative Compromise Ranking (ICOR) and Multi- Attribute Utility Theory (MAUT) are a well-known multi-criteria decision making methods [7]. MAUT approach uses a function called Utility. Decision maker assigns a utility value to each alternative. This utility reflects the alternatives importance to the decision. The utility values can range from 1 to 10 , where 1 represents the lowest importance and 10 the highest. Decision maker gives a score to each of alternatives for each attribute. The final ranking value of the alternative is calculated as the sum of each alternative's score for each attribute multiplied by the weight of that attribute. The alternative with the best value of the aggregated function is considered as the best of the alternatives. MAUT method is applied in all types of problems which have significant amount of uncertainty, such as financial, energy management problems, etc. Reference [1] underlined AHP method as simpler form of MAUT. The utility function is calculated using the technique based on pairwise comparisons. The weights for the criteria and the relative performance scores for each of the alternatives on each criterion are determined by this technique. The relative scale from 1 to 9 is used to measure importance between two criteria. The higher number means the chosen criterion is considered more important than other criterion being compared with. The scores are assigned for each alternative option for each criterion using normalized pairwise comparisons matrix of identified criteria. ICOR is the third method described in this paper. It defines a narrow set of possible solutions closest to the ideal solution. The weight is assigned to each criterion and represents its relative importance. The compromise solution can be established by different strategies. The example is the strategy of "the majority of criteria". This strategy is demonstrated in our previous research [13].

We now propose to use two different group decision making methods to rank a finite set of 
alternatives. Arithmetic-mean and geometric-mean as aggregation methods are introduced. We want to present some approaches to creating the preference structure of alternatives by simply assigning ordinal ranks to the different alternatives. These methods will be further discussed throughout the following sections.

Choosing a Cloud provider is also a multiple attributes decision making problem. An increasing number of companies are offering variety of Cloud Computing services to enterprises. Amazon Web Services and Salesforce.com are regarded as the pioneers of Cloud Computing. Google, Microsoft, IBM, GoGrid, and Rackspace have also entered the Cloud market. So many Cloud providers create healthy competition among providers to meet the Service Level Agreement (SLA) requirements. The provider is obligated to meet performance objectives defined by the SLA metrics. Despite different protecting strategies and technologies for solving security and privacy problems, the service agreement signed with service provider becomes the only guarantee. It is necessary to assess security risks implied by the act of embedding resources within the CC environment. A major consideration should be data security and data availability of company's critical data in the Cloud. The location of the data centers and servers must be known to ensure the security regulations are followed. Cloud provider should have very limited access to sensitive and critical data. These added security features can result in higher providers' prices.

The extended general model for Cloud adoption is presented in Figure 1. The extended model is consist of two new aggregation methods. The Figure 1 shows seven key factors and brief explanation of each, with the aim to support appropriate analysis of different aspects.

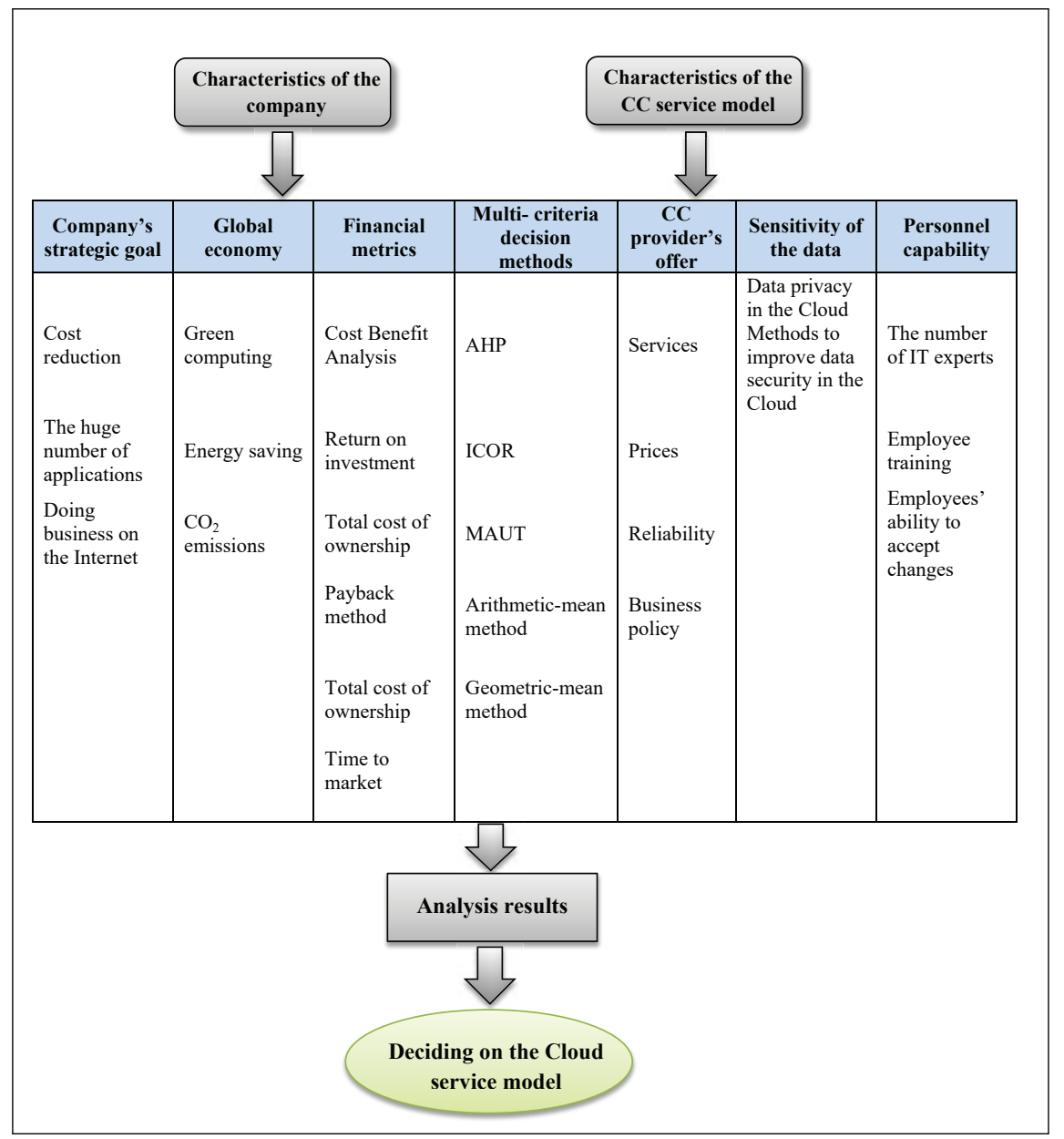

Figure 1: Extended general model for Cloud adoption 


\section{Methods and Results}

\subsection{Decision- making using AHP, MAUT and ICOR Methods}

In this section, we briefly present the results from methods that previously have been applied to ranking different Cloud services [13]. Experts from the academic and the business world provide criteria weights and take part in ranking different alternatives. Table 1 summarized the criteria considered in our research, while Table 2 summarized the alternatives.

\begin{tabular}{|l|}
\hline Criteria \\
\hline Data security \\
\hline Data availability \\
\hline The monthly average price \\
\hline Ease of use \\
\hline Flexibility \\
\hline Consumer Scope of Control \\
\hline The level of customization \\
\hline The level of Cloud provider support \\
\hline The risk of changing Cloud provider \\
\hline Overall business growth \\
\hline Company size \\
\hline Number of IT experts \\
\hline
\end{tabular}

Table 1: Defined set of criteria

\begin{tabular}{|l|}
\hline Alternatives \\
\hline Software as a Service \\
\hline Platform as a Service \\
\hline Infrastructure as a Service \\
\hline Network as a Service \\
\hline Storage as a Service \\
\hline Database as a Service \\
\hline Security as a Service \\
\hline Integration as a Service \\
\hline Management as a Service \\
\hline Testing as a Service \\
\hline Information as a Service \\
\hline Communication as a Service \\
\hline Monitoring as a Service \\
\hline
\end{tabular}

Table 2: Defined set of alternatives

The methods results are presented in Table 3. From these results, we can conclude that fifth alternative, Storage as a Service model, is the alternative with the largest utility values. The flexibility and ease of use may be the reason for the highest score. On the other hand, it has a low impact on business's growth. The alternative with the lowest utility values is Platform as a Service. With PaaS, consumer needs to put in development effort to create and test applications 
and to have control over the deployed applications. The problem appears in understanding of who is responsible for which areas of CC architecture.

The resulting weights of the AHP method show Software as a Service model as the alternative with the highest weighted preference. Software as a Service model is the most suitable model because it provides significant efficiencies in cost and Cloud provider is fully responsible for all components of $\mathrm{CC}$ architecture. However, decision makers cannot neglect existing security challenges. The lowest weighted preference has Platform as a Service model.

Using IKOR method decision makers get the solution that is the closest to the ideal. The alternative option that is the closest to the ideal is Software as a Service model, while on the last position in ranking list is Network as a Service model. The disadvantage of creating the specific virtual network is the need to control the devices from the Cloud.

\begin{tabular}{|l|c|c|c|}
\hline Alternatives & MAUT method & AHP method & ICOR method \\
\hline Software as a Service & 8.105 & 0.110 & 1 \\
\hline Platform as a Service & 6.393 & 0.057 & 11 \\
\hline Infrastructure as a Service & 6.662 & 0.062 & 10 \\
\hline Network as a Service & 6.424 & 0.071 & 13 \\
\hline Storage as a Service & 8.447 & 0.106 & 2 \\
\hline Database as a Service & 7.328 & 0.065 & 6 \\
\hline Security as a Service & 6.982 & 0.058 & 9 \\
\hline Integration as a Service & 7.648 & 0.082 & 3 \\
\hline Management as a Service & 6.994 & 0.058 & 7 \\
\hline Testing as a Service & 8.152 & 0.088 & 4 \\
\hline Information as a Service & 7.351 & 0.071 & 18 \\
\hline Communication as a Service & 7.129 & 0.069 & 12 \\
\hline Monitoring as a Service & 7.204 & 0.092 & 5 \\
\hline
\end{tabular}

Table 3: The final ranking of the alternative options using MAUT, AHP and ICOR method

\subsection{Group decision making using Aggregation Methods}

In many group decision making problems, individual preferences on a given set of alternatives are obtained from several decision makers and then these individual preferences are aggregated to a single collective preference. For group problem solving, the individuals participating in making a group decision assign ranks to the same finite set of alternatives. The idea is to harmonize opinions of the participants in the group in order to select the most acceptable alternative for the whole group.

We will use in our approach two types of preference aggregation methods for harmonization of alternatives rankings [2]. These methods require, as the input data, individual preferences on a same set of alternatives and derive the consensus ranking that minimized disagreement among a group. They are considered as a single criterion analysis, since the individuals compare alternatives directly. Here we present the approach to implement these methods using alternatives rankings obtained by the previous demonstrated decision making methods in [13]. Each method's ranking is combined to produce an overall assessment. In this way we critically evaluate results of previous research. Moreover, it reduces subjectivity of assigning individual preference values to alternatives by decision makers.

First method is arithmetic-mean method based on arithmetic mean of the ranking made by all decision makers. The overall preference of an alternative is estimated by arithmetic mean. 
The result is an aggregated value that is between maximum and minimum value. The most acceptable alternative has the lowest average overall rate. Geometric-mean method is similar to arithmetic-mean method. It uses the geometric mean of individual ranks instead of the simple arithmetic mean to determine the average rank. The alternative with the lowest value is the most acceptable. A geometric mean is always less than or equal to an arithmetic mean. It tends to reduce the effect of very high and very low values, which helps in eliminating extreme values (outliers).

First we define individual ordering matrix of alternatives. Rows of a matrix relate to the alternatives, columns relate to methods results. Elements of individual ordering matrix represent alternative assigned rank for a method. The elements are shown in Table 4.

\begin{tabular}{|l|l|l|l|}
\hline Alternatives & $\begin{array}{l}\text { AHP method } \\
\text { ranking }\end{array}$ & $\begin{array}{l}\text { MAUT method } \\
\text { ranking }\end{array}$ & $\begin{array}{l}\text { ICOR method } \\
\text { ranking }\end{array}$ \\
\hline Software as a Service & 1 & 3 & 1 \\
\hline Platform as a Service & 13 & 13 & 11 \\
\hline Infrastructure as a Service & 10 & 11 & 10 \\
\hline Network as a Service & 7 & 12 & 13 \\
\hline Storage as a Service & 2 & 1 & 2 \\
\hline Database as a Service & 9 & 6 & 6 \\
\hline Security as a Service & 12 & 10 & 9 \\
\hline Integration as a Service & 5 & 4 & 3 \\
\hline Management as a Service & 11 & 9 & 7 \\
\hline Testing as a Service & 4 & 2 & 4 \\
\hline Information as a Service & 6 & 5 & 8 \\
\hline Communication as a Service & 8 & 8 & 12 \\
\hline Monitoring as a Service & 3 & 7 & 5 \\
\hline
\end{tabular}

Table 4: Individual ordering matrix of alternatives

There were no strong differences between the results of arithmetic-mean and geometricmean methods. Consensus is achieved for the most acceptable alternative and the next ranked alternative. Software as a service model and Storage as a service model is ranked at first and second place respectively. These alternatives dominated according to all three decision making methods. MAUT method ranked the Software as Service alternative in third place, while AHP and ICOR method elected this alternative as the most favorable Cloud service model. The reason for this result might be the significant reduction in IT cost and ultimately the provider is responsible for maintaining the software. We want to underline the slight difference in geometric mean value of these two alternatives. Moreover, two alternatives have the same average value of rankings. Table 5 reports arithmetic mean and geometric mean value of an alternative.

Furthermore, there is significant disagreement over the alternatives which are ranked the lowest. In these methods, Platform as a Service is ranked as the worst solution. The rank order of Security as a Service, Network as a Service, and Infrastructure as a Service, alternatives differs. It shows higher or lower score level of these alternatives in one of the previous decision making methods. Infrastructure as a Service alternative is valued considerably higher by MAUT method, while Security as a Service is valued considerably lower by AHP method. 


\begin{tabular}{|l|c|c|}
\hline Alternatives & Arithmetic mean value & Geometric mean value \\
\hline Software as a Service & 1.667 & 1.442 \\
\hline Platform as a Service & 12.333 & 12.296 \\
\hline Infrastructure as a Service & 10.333 & 10.323 \\
\hline Network as a Service & 10.667 & 10.298 \\
\hline Storage as a Service & 1.667 & 1.587 \\
\hline Database as a Service & 7.000 & 6.868 \\
\hline Security as a Service & 10.333 & 10.260 \\
\hline Integration as a Service & 4.000 & 3.915 \\
\hline Management as a Service & 9.000 & 8.849 \\
\hline Testing as a Service & 3.333 & 3.175 \\
\hline Information as a Service & 6.333 & 6.214 \\
\hline Communication as a Service & 9.333 & 9.1588 \\
\hline Monitoring as a Service & 5.000 & 4.718 \\
\hline
\end{tabular}

Table 5: Arithmetic-mean method and geometric-mean method results

\section{Conclusion}

Cloud Computing is a technology that surely transforms the way corporate IT services are delivered and managed. Decision to migrate existing services to the Cloud can be complicated as selecting the most favorable Cloud service model is is a rather complex process. The paper described the characteristics of different Cloud services with the aim of helping decision makers to fully understand organizational benefits and drawbacks of CC. The focus was on developing general model for Cloud adoption with the aim to support decision makers to investigate Cloud adoption decisions and to make the right choice.

The paper is an extension of [13] where different Cloud service models are evaluated using three multi criteria decision making methods, in particular AHP, ICOR and MAUT methods. The criteria preference values have been assigned by the authors after discussions with experts from different stakeholder groups. The final ranking of the alternative options for all three methods were reported. In this paper we thoroughly evaluated seven key factors of general model for Cloud adoption. Because most of the pros and cons of Cloud are unquantifiable, it is important that decision makers view Cloud adoption project from different perspectives in order to fully understand Cloud services. Furthermore, decision makers have to compare the cost of different Cloud providers, deployment options and usage scenarios. By using decision making methods, decision makers can rate and apply weights to quality attributes of different Cloud service models, based on their preferences. In the present paper we have introduced some other decision making methods to show another view of ranking Cloud services. The general model for Cloud adoption is extended with two new aggregation ranking methods for harmonization of alternatives ranking for group problem solving. The feasibility and usefulness of the application of arithmetic-mean method, and geometric-mean method, have been demonstrated. The new approach used alternatives rankings obtained by the previous demonstrated decision making methods. The results have shown that Software as a service model and Storage as a service model dominated according to not just arithmetic-mean method, but also geometric-mean method confirmed this. Furthermore, results proved Platform as a Service model as alternative with the worst preferences.

Therefore, we can highlight that consumers prefer Cloud service models with low risk solution implementation. In all demonstrated methods, Platform as a Service is ranked as one of the worst 
solution. This is due to the fact that the developers must work within the constraints of the platform. We can conclude that factors like: cost, ease of use and disclosed scope of controls between provider and consumer, are the main factors which determine the prioritization of Cloud service models. Data security and privacy protection issues did not play a key role in selecting right Cloud service model as we expected. The findings of this study are a base for a future work consisting in the concrete case study implementations. Certainly there may be additional factors which may have not been considered within this paper. It is also possible to evaluate other group decision making methods and types of preference aggregation methods for harmonization of alternatives rankings.

\section{Bibliography}

[1] Alexander, M. (2012); Decision-Making Using the Analytic Hierarchy Process ( AHP ) and JMP (R) Scripting Language, 2012 The SouthEast SAS Users Group Conference (SESUG), SESUG Inc.

[2] Alfares, H. K.; Duffuaa, S. O.(2008); Determining Aggregate Criteria Weights From Criteria Rankings By a Group of Decision Makers, International Journal of Information Technology \& Decision Making, 7(4): 769-781.

[3] Buyya, R. et al (2008), Cloud computing: Principles and paradigms, 8: 3-121.

[4] Carbon Disclosure Project 2011, Carbon Disclosure Project Study 2011 Cloud ComputingThe IT Solution for the 21st Century, Verdantix, White Paper.

[5] Costa, P.; Migliavacca, M.; Wolf, A. L. (2012); NaaS: Network-as-a-Service in the Cloud,2nd USENIX Workshop on Hot Topics in Management of Internet, Cloud, and Enterprise Networks and Service, San Jose, CA, USA.

[6] Cruz, Z.; Fernández-Alemán, J.; Toval A. (2015); Security in cloud computing: A mapping study, Computer Science and Information Systems, 12(1): 161-184.

[7] Figueira, J.; Greco, S.; Ehrogott, M. (2005),Multiple Criteria Decision Analysis: State of the Art Surveys, Springer Science+Business Media, Inc., 27-406.

[8] Hashizume, K. et al (2013); An analysis of security issues for cloud computing, Journal of Internet Services and Application, 4(5): 1-13.

[9] Kornevs, M.; Minkevica, V.; Holm, M. (2012); Cloud Computing Evaluation Based on Financial Metrics, Information Technology and Management Science, 15(1): 87-92.

[10] Mell, P.; Grance, T. 2011, The NIST Definition of Cloud Computing, NIST Special Publication 800-145, National Institute of Standards and Technology Gaithersburg.

[11] Messmer, E. (2013), Gartner: Cloud-based security as a service set to take off, http://www.networkworld.com/article/2171424/data-breach/gartner-cloud-based-securityas-a-service-set-to-take-off.html, Information Technology and Management Science

[12] Pajić, A.; Pantelić, O.; Stanojević B. (2014); Representing IT Performance Management as Metamodel, International Journal of Computers Communications $\&$ Control, 9(6): 758-767. 
[13] Pantelić, O.; Pajić, A.; Nikolić, A.; (2016); Analysis of available cloud computing models to support cloud adoption decision process in an enterprise, Computers Communications and Control (ICCCC), 2016 6th International Conference on, IEEE Xplore, e-ISBN:978-1-50901735-5, doi: 10.1109/ICCCC.2016.7496751, 135-139.

[14] Patel, K. H. et al (2012); Tradeoffs between performance and security of cryptographic primitives used in Storage-as-a-Service for cloud computing, Proceedings of the CUBE International Information Technology Conference, New York, NY, USA.

[15] Pătraşcu, A.; Patriciu, V. V. (2015); Logging for Cloud Computing Forensic Systems Related work, International Journal of Computers Communications 85 Control, 10(2): 222-229.

[16] Sharma, S. (2015), Evolution of as-a-Service Era in Cloud, http://web.cs.iastate.edu/ ${ }^{\sim}$ sugamsha/articles/Evolution\%20of\%20as-aService\%20Era\%20in\%20Cloud.pdf

[17] Simmonds, M. (2009), Information as a service: A smarter way to SOA success, IBM Software Group, 1-19.

[18] Waschke, M. (2004), IT Management-As-A-Service, CA Technologies, Whitepaper, no. 451. 